\title{
A Meta-analysis of the Value of vWF in the Diagnosis of Liver Cirrhosis with Portal Hypertension
}

\author{
Xiang-Chun Ding ${ }^{\# 1}$, Wan-Long Ma ${ }^{\# 1}$, Ming-Kun Li ${ }^{\# 2}$, Shuai-Wei Liư ${ }^{1}$, Xiao-Yan Liu ${ }^{1}$, \\ Long $\mathrm{Hai}^{1}$, Xia Luo ${ }^{1}$, Yan-Chao Hu${ }^{1}$ and Li-Na Ma*1 \\ ${ }^{1}$ Department of Infectious Disease, General Hospital of Ningxia Medical University, Yinchuan, Ningxia, China; \\ ${ }^{2}$ Department of Gastroenterology, Municipal Hospital Affiliated to Xuchang, Xuchang, Henan, China
}

\section{Abstract}

Background and Aims: Studies have indicated that serum von Willebrand factor (VWF) has a positive correlation with hepatic venous pressure gradient. However, information on the value of VWF in the diagnosis of liver cirrhosis with portal hypertension has been lacking. The purpose of this meta-analysis was to assess the value of VWF in the diagnosis of liver cirrhosis with portal hypertension. Methods: Studies that analyzed the sensitivity, specificity, diagnostic odds ratio combined with likelihood ratios and test for heterogeneity of VWF in the diagnosis of liver cirrhosis with portal hypertension were found in the Cochrane Library, Ovid, VOS-SCI, CNKI, PubMed, Medline, EMBASE, CMB and Wanfang databases. In the end, the data was used to draw the summary receiver operating characteristic curve and to calculate the area under the curve. Results: Four studies involving 662 patients were analyzed. The results showed that serum vWF in liver cirrhosis with portal hypertension were significantly higher than in those without portal hypertension. Sensitivity combined was 0.823 (95\% CI: $0.788,0.855)$. Specificity combined was 0.782 (95\% CI: $0.708,0.845)$. +LR combined was 3.777 (95\% CI: 2.794, 5.107). -LR combined was 0.221 (95\% CI: $0.180,0.272$ ). Diagnostic odds ratio combined was 18.347 ( $95 \% \mathrm{CI}$ : $11.725,28.708)$. The area under the curve was 0.8896 . Conclusions: Serum vWF can be used as an effective and feasible method for noninvasive diagnosis of liver cirrhosis with portal hypertension. However, further studies are still needed to evaluate the severity of liver cirrhosis with portal hypertension.

Citation of this article: Ding XC, Ma WL, Li MK, Liu SW, Liu XY, Hai $L$, et al. A meta-analysis of the value of vWF in the diagnosis of liver cirrhosis with portal hypertension. J Clin Transl Hepatol 2019;7(1):3-8. doi: 10.14218/JCTH.2018.00036.

Keywords: Liver cirrhosis; Portal hypertension; HVPG; vWF.

Abbreviations: AUC, area under the curve; CI, confidence interval; DOR, diagnostic odds ratio; HVPG, hepatic venous pressure gradient; LR, likelihood ratio; $\mathrm{PH}$, portal hypertension; SEN, sensitivity; SPE, specificity; SROC, summary receiver operating characteristic; vWF, von Willebrand factor.

Received: 10 June 2018; Revised: 9 September 2018; Accepted: 14 September 2018

\#These authors contributed equally to this work.

* Correspondence to: Dr. Li-Na Ma, Department of Infectious Disease, General Hospital of Ningxia Medical University, Yinchuan, Ningxia 750004, China. Tel: +86-13619511758, E-mail: 13619511758@163.com

\section{Introduction}

Liver cirrhosis is a chronic liver disease, which is caused by one or more factors, such as virus infection, drugs and toxicants, heavy drinking, metabolic disorders, liver hemodynamic disorders, high fat diet, and so on. These factors have a long-term effect on liver tissue, leading to diffuse fibrosis of the liver, pseudo lobules, and regenerative nodules. ${ }^{1}$ It is the final outcome of many kinds of liver diseases.

Cirrhosis combined with portal hypertension (HP) is an important manifestation of cirrhosis progression to decompensation. Complications associated with $\mathrm{PH}$ severely reduce the prognosis and survival rate of patients with cirrhosis. Therefore, the early diagnosis and effective treatment of $\mathrm{PH}$ are particularly important. The study of the effective evaluation index and early intervention of $\mathrm{PH}$ are expected to improve the life cycle and quality-of-life of patients with the disease.

The current hepatic venous pressure gradient (HVPG) is still the gold standard for evaluating portal pressure. ${ }^{2,3}$ Normal portal pressure is defined as the range of 1 to 5 $\mathrm{mmHg}$, according to HVPG. When the HVPG range of 6 to 9 $\mathrm{mmHg}$ is reached, $\mathrm{PH}$ is defined as subclinical $\mathrm{PH}$, whereas HVPG $\geq 10 \mathrm{mmHg}$ is diagnosed as clinically significant $\mathrm{PH} .{ }^{2,3}$ At this time, the risk of cirrhosis-related complications increases significantly. ${ }^{3}$ Yet, HVPG is a kind of invasive operation characterized by high risk and needs for advanced equipment, high level of operation skill of medical workers, high price and difficult follow-up, all of which limit its universal application in China. It is still necessary to find a simple and noninvasive method for predicting $\mathrm{PH}$ in cirrhosis to guide the diagnosis and treatment of it.

von Willebrand factor (VWF), as a marker of vascular endothelial damage, is a macromolecular glycoprotein, mainly composed of endothelial cells and bone marrow megakaryocytes. ${ }^{4}$ The plasma levels of vWF are related to stress, vascular endothelial damage, activation of platelets, and hepatic sinusoidal endothelial system clearance. ${ }^{5}$ In addition, as a component of the extracellular matrix, vWF has been shown to play an active role in regulating vascular proliferation, liver injury and repair, and to play an important role in the occurrence and development of cirrhosis with $\mathrm{PH} .{ }^{6,7}$ Studies have shown that serum vWF levels and HVPG in patients with cirrhosis and $\mathrm{PH}$ have a positive correlation and can predict clinical outcome in such patients. ${ }^{6,7}$ However, the value of vWF in the diagnosis of $\mathrm{PH}$ in liver cirrhosis are not yet clear. 
Based on this background, to explore the value of VWF in the diagnosis of $\mathrm{PH}$ in liver cirrhosis, we made a metaanalysis of the published studies of VWF in the diagnosis of $\mathrm{PH}$ in liver cirrhosis. The databases of Cochrane Library, Ovid, VOS-SCI, CNKI, PubMed, Medline, EMBASE, CMB and Wanfang were searched. We discovered that serum vWF could be used as an effective and feasible method for noninvasive diagnosis of liver cirrhosis with $\mathrm{PH}$. However, further studies are still needed to evaluate the severity of liver cirrhosis with $\mathrm{PH}$.

\section{Methods}

\section{Literature search}

The literature available in the Cochrane Library, Ovid, VOS-SCI, CNKI, PubMed, Medline, EMBASE, CMB and Wanfang databases was searched. The subject words and reference words used were determined by the subject word list of Medicine (commonly known as MeSH terms) and the Chinese version of key words. According to the characteristics of the different databases, combinations of the corresponding subject words and reference words were used to search. The reference literature was searched at the same time.

\section{Inclusion and exclusion criteria}

Inclusion criteria for the meta-analysis were as follows. (1) Prospective study, continuity research or retrospective study. (2) Diagnosis of cirrhosis based on pathological examination or clinical diagnosis according to physical signs, ultrasound, CT and biochemical indexes. It included hepatic sinusoidal obstruction syndrome, chronic alcoholic liver disease, autoimmune liver disease, hereditary metabolic liver disease, liver cirrhosis caused by various viral infections, combined esophageal and gastric variceal bleeding, intractable ascites, hypersplenism, liver cancer, and patients with $\mathrm{PH}$ that had persistent treatment. The diagnosis of $\mathrm{PH}$ was made on the basis of HVPG $\geq 10 \mathrm{mmHg}$. (3) All subjects had measures of serum vWF. (4) The study could have listed quadruple tabular form.

Patient populations were excluded if they featured: (1) increasing serum VWF not due to liver cirrhosis; (2) diagnosis of PH not based on HVPG; (3) data about the serum VWF and HVPG unable to be extracted.

\section{Literature collection and selection}

The search was based on the search strategy described in the previous session. First, according to the topic of the literature, we eliminated the literature which was obviously inconsistent with the inclusion criteria. Secondly, we read the summary part of the literature, and suspicious literature was temporarily left. Finally, we read the literature carefully, according to the inclusion and exclusion criteria to ultimately determine the literature selected.

\section{Quality evaluation}

The QUADAS tool was used to assess the methodological quality of the selected studies. This tool is a validated quality checklist containing 14 items that address the most important sources of bias and variation in diagnostic accuracy studies. ${ }^{8}$ The detailed explanations of the 14 items are elaborated in Table 3. The reviewers who assessed and extracted data were well-trained in the use of the QUADAS checklist. Each item in the checklist was categorized as "Yes" for low risk of bias, "No" for high risk of bias, or "Unclear" if there was insufficient information to make an unbiased judgment. 8

\section{Statistical analysis}

The data extracted from the included literature are listed in a four-fold table. First, Meta software was used to analyze the sensitivity (SEN), specificity (SPE) and 95\% confidence interval (CI) of VWF in the diagnosis of $\mathrm{PH}$ in liver cirrhosis. Then, Meta-DiSc1.4 software was used to analyze SEN, SPE, positive (+) likelihood ratio (LR), negative (-)LR, diagnostic odds ratio (DOR) combined and test for heterogeneity. We considered significant heterogeneity having been met when the $X^{2}$ value was within the $10 \%$ level of significance $(p<0.10)$. In the end, the data was used to draw summary receiver operating characteristic (SROC) curves and to calculate the area under the curve (AUC).

\section{Results}

\section{An overview of and quality analysis of the literature included}

A total of 27 studies were identified and screened for retrieval by using the strategies described above. After screening the title or abstract, 11 studies were excluded and 14 were retrieved and subjected to detailed evaluation. By adhering to the inclusion criteria, 10 of those studies were excluded. Finally, 4 cohort studies ${ }^{6,9-11}$ were chosen for inclusion in the metaanalysis, which comprised a total of 662 patients (Fig. 1).

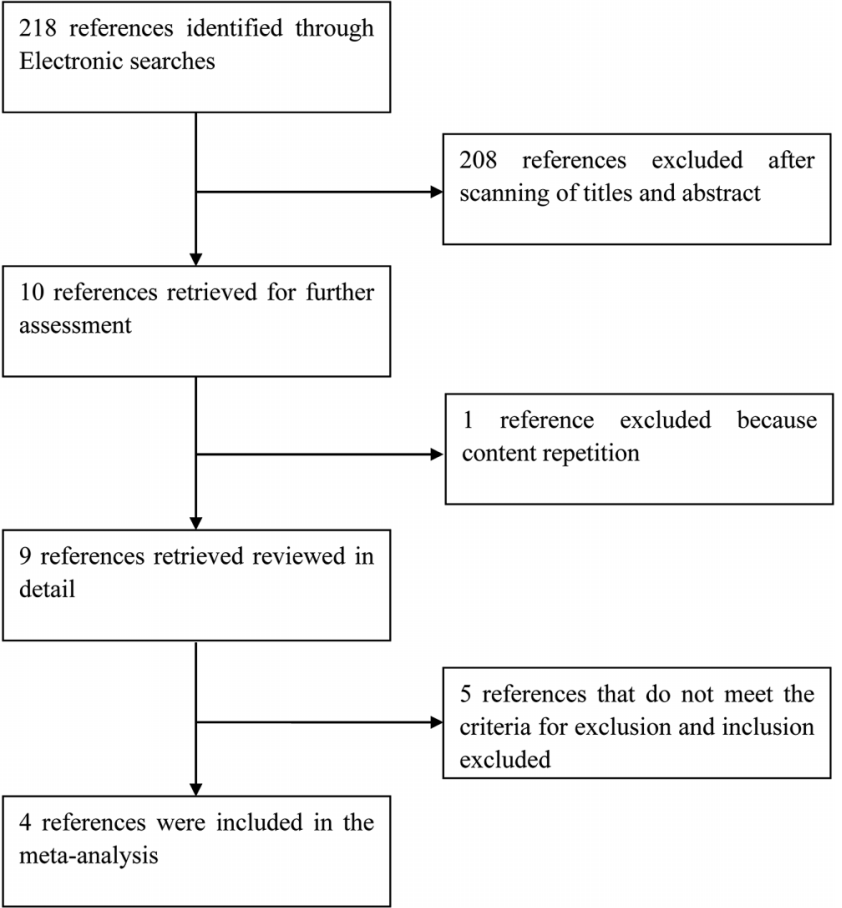

Fig. 1. Literature search flow sheet. 
Ding X.C. et al: vWF and portal hypertension

Table 1. The basic information of the literature included

\begin{tabular}{|c|c|c|c|c|}
\hline Study & Monika Ferlitsch & Stephanie Hametner & Hao Wu & M. Homonclik \\
\hline Year of publication & 2012 & 2016 & 2015 & 2017 \\
\hline Sample size & 286 & 236 & 60 & 81 \\
\hline Male/Female & $201 / 65$ & $170 / 66$ & $43 / 17$ & $67 / 14$ \\
\hline Age & $55(48-62)$ & $57.9(50-60)$ & $49(45-52)$ & 54 \\
\hline Etiology & - & $\begin{array}{l}\text { ALD 93; HCV 67; NASH 29; } \\
\text { other 19; unknown } 28\end{array}$ & $\mathrm{HBV}$ & $\begin{array}{l}\text { ALD, HCV, } \\
\text { cryptogenic }\end{array}$ \\
\hline $\operatorname{CPS}(\mathrm{A} / \mathrm{B} / \mathrm{C})$ & $48 / 104 / 34$ & $140 / 56 / 18$ & $28 / 19 / 13$ & - \\
\hline Compensation & 189 & 136 & - & - \\
\hline Decompensation & 97 & 100 & - & - \\
\hline
\end{tabular}

According to the Review Manager 5.2 diagnostic test system evaluation criteria, we needed to independently evaluate the research characteristics and retrieve relevant information, including sample size, sample characteristics and research type. The basic characteristics of each of the 4 studies are listed in Table 1 . Of the 4 studies, 1 study $^{9}$ was composed of Chinese and the other $3^{6,10,11}$ were composed of Australians. The population size for each of the studies ranged from 60 to 286 . The mean age ranged from 49 to 57.9 years old. The percentage of males ranged from $70.3 \%$ to $82.7 \%$.
According to the 14 criteria of QUADAS used evaluate the quality of the literature, the literature of Monika Ferlitsch and Stephanie Hametner have the highest quality, while that of M. Homonclik has the lowest quality (Table 2).

\section{The serum vWF expression increased significantly in cirrhotic patients with $\mathbf{P H}$}

The 4 studies are based on the HVPG measurement of liver cirrhosis, and the patients were divided into $\mathrm{PH}$ group and group without $\mathrm{PH}$. Then, the serum vWF was detected. By comparing the serum vWF (Table 3 ) in the group without $\mathrm{PH}$

Table 2. Quality assessment

\begin{tabular}{|c|c|c|c|c|}
\hline Study & $\begin{array}{l}\text { Monika } \\
\text { Ferlitsch }\end{array}$ & $\begin{array}{l}\text { Stephanie } \\
\text { Hametner }\end{array}$ & $\begin{array}{l}\text { Hao } \\
\text { Wu }\end{array}$ & $\begin{array}{l}\text { M. } \\
\text { Homonclik }\end{array}$ \\
\hline $\begin{array}{l}\text { Was the spectrum of patients representative of the patients who will } \\
\text { receive the test in practice? }\end{array}$ & Yes & Yes & Yes & Yes \\
\hline Were selection criteria clearly described? & Yes & Yes & Yes & Yes \\
\hline Is the reference standard likely to correctly classify the target condition? & Yes & Yes & Yes & Yes \\
\hline $\begin{array}{l}\text { Did patients receive the same reference standard regardless of the index } \\
\text { test result? }\end{array}$ & Yes & Yes & Yes & Yes \\
\hline Was the reference standard independent of the index test? & Yes & Yes & Yes & Yes \\
\hline $\begin{array}{l}\text { Were the reference standard results interpreted without knowledge of the } \\
\text { results of the index test? }\end{array}$ & Yes & Yes & Yes & Yes \\
\hline $\begin{array}{l}\text { Were the same clinical data available when test results were interpreted } \\
\text { as would be available when the test is used in practice? }\end{array}$ & Yes & Yes & Yes & Yes \\
\hline Were withdrawals from the study explained? & Yes & Yes & Yes & Yes \\
\hline $\begin{array}{l}\text { Were the index test results interpreted without knowledge of the results of } \\
\text { the reference standard? }\end{array}$ & No & No & No & No \\
\hline $\begin{array}{l}\text { Is the time period between reference standard and index test short } \\
\text { enough to be reasonably sure that the target condition did not change } \\
\text { between the two tests? }\end{array}$ & Yes & Yes & Unclear & Unclear \\
\hline $\begin{array}{l}\text { Did the whole sample or a random selection of the sample, receive } \\
\text { verification using a reference standard of diagnosis? }\end{array}$ & Yes & Yes & Yes & Yes \\
\hline $\begin{array}{l}\text { Was the execution of the index test described in sufficient detail to permit } \\
\text { replication of the test? }\end{array}$ & Yes & Yes & Yes & No \\
\hline $\begin{array}{l}\text { Was the execution of the reference standard described in sufficient detail } \\
\text { to permit its replication? }\end{array}$ & Yes & Yes & Yes & No \\
\hline Were uninterpretable/ intermediate test results reported? & Yes & Yes & Yes & No \\
\hline
\end{tabular}


Table 3. The serum levels of vWF in the included studies

\begin{tabular}{llll}
\hline \multirow{2}{*}{ vWF } & $\begin{array}{l}\text { Cirrhosis without portal hypertension } \\
(\mathrm{HVPG}<10 \mathrm{mmHg})\end{array}$ & $\begin{array}{l}\text { Cirrhosis with portal hypertension } \\
(\mathrm{HVPG} \geq 10 \mathrm{mmHg})\end{array}$ & Cutoff \\
\hline Monika Ferlitsch & $197 \%(158 \%-228 \%)$ & $346 \%(275 \%-441 \%)$ & $241 \%$ \\
Stephanie Hametner & $200 \%(157 \%-236 \%)$ & $306 \%(227 \%-373 \%)$ & $226 \%$ \\
M. Homonclik & $210 \%(176 \%-243 \%)$ & $355 \%(322 \%-388 \%)$ & $264 \%$ \\
Hao Wu & $1240 \pm 470.3 \mathrm{mU} / \mathrm{mL}$ & $2430 \pm 760.3 \mathrm{mU} / \mathrm{mL}$ & $1510.5 \mathrm{mU} / \mathrm{mL}(231 \%)$ \\
\hline
\end{tabular}

\begin{tabular}{|c|c|c|c|c|c|c|c|c|}
\hline Stucly & TP & FP & $\mathbf{F N}$ & TN & Sensitivity $(95 \% \mathrm{Cl})$ & Specificity (95\% Cl) & Sensitivity $(95 \% \mathrm{Cl})$ & Specificity ( $95 \% \mathrm{Cl})$ \\
\hline HAO WU 2015 & 45 & 7 & 3 & 5 & $0.94[0.83,0.99]$ & $0.42[0.15,0.72]$ & $\rightarrow$ & $\longrightarrow$ \\
\hline M.Homonclik 2008 & 54 & 1 & 11 & 15 & $0.83[0.72,0.91]$ & $0.94[0.70,1.00]$ & $=$ & \\
\hline Monika 2012 & 180 & 14 & 30 & 61 & $0.86[0.80,0.90]$ & $0.81[0.71,0.89]$ & $\rightarrow$ & $\Rightarrow$ \\
\hline Stephanie 2016 & 150 & 11 & 48 & 27 & $0.76[0.69,0.82]$ & $0.71[0.54,0.85]$ & $-\frac{9}{9}$ & $-\frac{1}{1}$ \\
\hline
\end{tabular}

Fig. 2. Forest map of the meta-analysis.

and the $\mathrm{PH}$ group, the expression of serum vWF in the cirrhosis $\mathrm{PH}$ group was found to be significantly higher than that in the liver cirrhosis without PH group.

\section{Meta-analysis}

The 4 studies did not directly provide four-fold tables (i.e. true positive number, false positive number, false negative number and true negative number), but the effective data were extracted by statistical processing through the SEN and SPE of the positive and negative results obtained by the evaluation test method; in this way, the four-fold table was generated, ${ }^{12}$ and then RevMan5.2 software was used for meta-analysis. The SEN, SPE and $95 \% \mathrm{CI}$ were calculated (Fig. 2).

\section{Meta-analysis of diagnostic test in Meta-DiSc software}

Threshold effect: Testing heterogeneity is the key factor that clears the possible factors affecting the accuracy and determines whether the accuracy of different studies is appropriate. ${ }^{12}$ In the meta-analysis of diagnostic tests, the
A

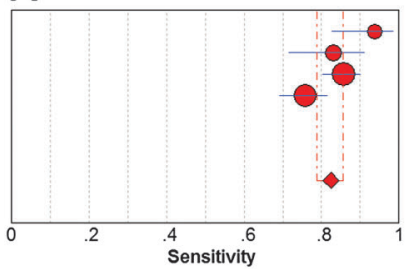

HAO WU M. Homonclik Monika Stephanie

Pooled Sensitivity $=0.82$ ( 0.79 to 0.86 ) Chi-square $=12.63 ; \mathrm{df}=3(\mathrm{p}=0.0055)$

Sensitivity

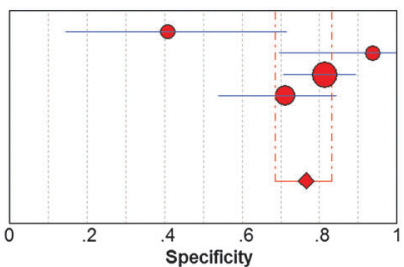

HAOWU

M. Homonclk

Monika

Stephanie

Pooled Specificity $=0.77$ (0.69 to 0.83 ) Chi-square $=12.04 ; \mathrm{df}=3(\mathrm{p}=0.0073)$ Inconsistency $($ (I-square $)=75.1 \%$
Sensitivity $(95 \% \mathrm{Cl})$ $\begin{array}{ll}0.94 & (0.83-0.99)\end{array}$ $0.83(0.72-0.91)$ $\begin{array}{ll}0.86 & (0.80-0.90) \\ 0.76 & (0.69-0.82)\end{array}$ $0.76 \quad(0.69-0.82)$
B

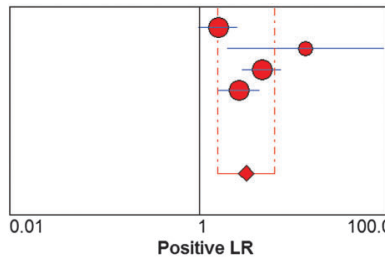

HAO WU $1.58(0.98-2.55)$ $\begin{array}{ll}13.29 & (1.99-88.95) \\ 4.59 & (2.85-7.39)\end{array}$ Stephanie $\quad 2.62(1.58-4.33)$

Random Effects Model Pooled Positive LR $=3.12$ (1.56 to 6.25$)$ Cochran- $Q=15.11 ; \mathrm{df}=3(\mathrm{p}=0.0017)$ Inconsistency (I-square) $=80.1 \%$ Tau-squared $=0.3556$

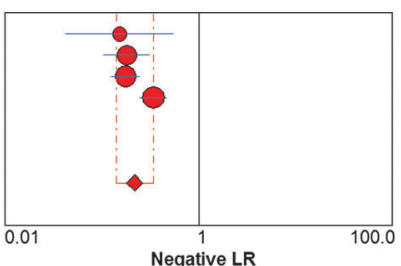

HAO WU M. Homonclik Monika Stephanie

Negative LR $(95 \% \mathrm{Cl}$ Specificity $(95 \% \mathrm{Cl})$ \begin{tabular}{l|l}
0.41 & $(0.15-0.72)$ \\
0.94 & $(0.70-1.00)$ \\
0.81 & $(0.71-0.89)$
\end{tabular} $0.81 \quad(0.71-0.89)$ $0.71 \quad(0.54-0.85)$

C

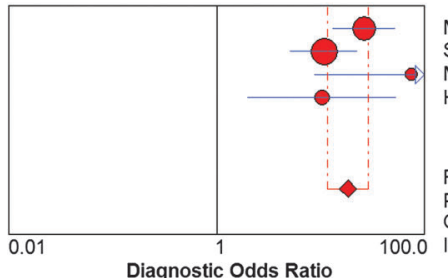

Monika Stephanie M. Homonclik HAO WU

Diagnostic OR $(95 \% \mathrm{Cl})$

Random Effects Mode Pooled Negative LR $=0.22$ ( 0.14 to 0.34 ) Cochran- $Q=9.65 ; d f=3(p=0.0218)$ Inconsistency $(1-$-square $)=68.9 \%$ Tau-squared $=0.1244$

Fixed Effects Mode

Pooled Diagnostic Odds Ratio $=18.35$ (11.73 to 28.7

Diagnostic Odds Ratio

Cochran- $Q=5.05 ; \mathrm{df}=3(\mathrm{p}=0.1683$

Fig. 3. Forest map of diagnostic odds ratio. 


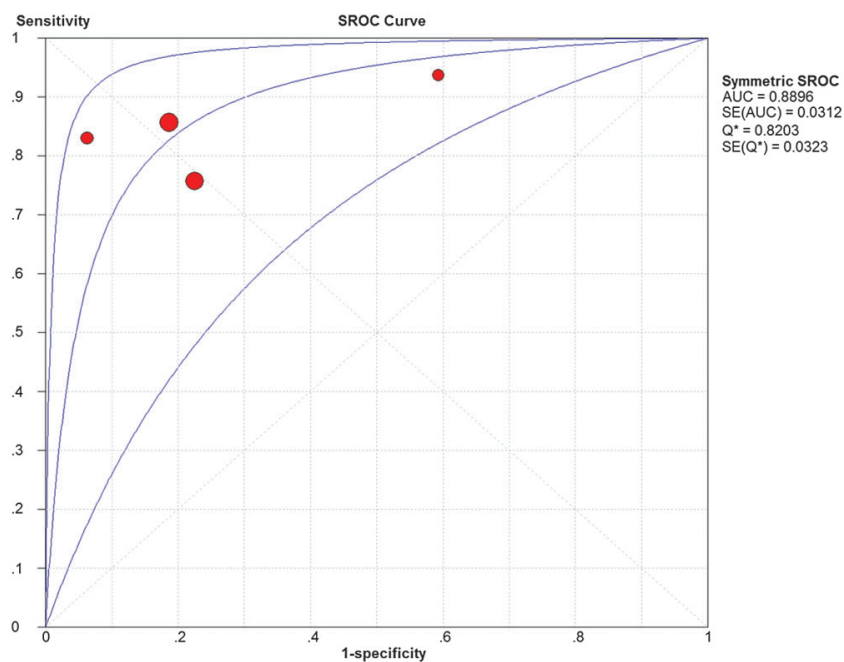

Fig. 4. Summary receiver operating characteristic curve.

threshold effect is one of the important reasons underlying heterogeneity. When there is a negative correlation between the logarithm of SEN and the logarithm of SPE (or the logarithm of SEN is positively correlated with the logarithm of 1- SPE), it indicates a threshold effect. In our test, the Spearman correlation coefficient between the logarithm of SEN and the logarithm of 1 - SPE was $0.400(r=0.400)$, indicting a positive correlation. But, $p=0.600(>0.1)$ indicated that there is no statistical significance, that is to say there was no threshold effect.

Combined effect sizes and discussed heterogeneity: Meta-DiSc1.4 software, which used the random effect model to analyze SEN, SPE, +LR, -LR (Fig. 3A-B), and DOR combined, drew a forest map of the DOR and test for heterogeneity (Fig. 3C). As shown in Figure 3C, the findings of $p=0.1683$, $I^{2}=40.6 \%, p>0.10$ and $I^{2}<50 \%$ indicated no heterogeneity.

Drawing of the SROC curve: The Mantel-Haenszel model of Meta-DiSc1.4 was used to combine the DOR and calculate the total SEN, SPE and 95\%CI. Then, the SROC curve was drawn and the AUC was calculated. Figure 4 shows the AUC of 0.8896 and $Q$ index of 0.8203 , indicating higher accuracy and better diagnostic efficiency.

\section{Discussion}

$\mathrm{PH}$ is an important sign of decompensation of liver cirrhosis. The increase of portal pressure can cause complications, such as upper gastrointestinal hemorrhage, massive ascites, splenomegaly, hypersplenism, hepatic encephalopathy, hepatorenal syndrome, and hepatopulmonary syndrome. ${ }^{3}$ It is an important pathophysiological link to continuously accelerating liver function decline or even failure. Therefore, the severity of $\mathrm{PH}$ is also an important indicator to predict the risk of death in patients with cirrhosis. ${ }^{13}$

HVPG is used as a gold standard for the diagnosis of cirrhosis with $\mathrm{PH} .{ }^{3,10,14}$ The study showed that the risk of upper gastrointestinal bleeding, massive ascites, spontaneous peritonitis, hepatic encephalopathy, liver cancer and other related complications increased significantly when HVPG was more than $10 \mathrm{mmHg} .2,3,6,7$ However, HVPG is an invasive method, associated with high cost and needs of advanced equipment and technical skills for diagnosis of $\mathrm{PH}$, so it is difficult to measure in clinic. Therefore, it is necessary to find noninvasive methods for detecting $\mathrm{PH}$, and such will have a very important role in early diagnosis and prediction of significance.

$v W F$, which is a marker of vascular endothelial damage, plays an important role in the occurrence and development of cirrhosis with $\mathrm{PH}^{6,7}$ Studies showed that the serum vWF levels were significantly positively correlated with HVPG in cirrhosis patients with $\mathrm{PH}$ and were able to predict clinical outcome of cirrhosis patients with $\mathrm{PH} .{ }^{6,7,9-11}$ Abdelmoneim et al. and Ferlitsch et al. found that vWF can be used as a potential new biomarker and noninvasive serum marker to diagnosis cirrhosis $\mathrm{PH} .6,10,14$ However, the value of vWF levels in the diagnosis of $\mathrm{PH}$ in liver cirrhosis is not yet clear.

In this study, to explore the value of VWF in the diagnosis of $\mathrm{PH}$ of cirrhosis, we searched for relevant literature in the Cochrane Library, Ovid, VOS-SCI, CNKI, PubMed, Medline, EMBASE, CMB and Wanfang databases and extracted data to analyze the correlation between HVPG and serum VWF in patients with cirrhosis and $\mathrm{PH}$. A total of 4 studies, comprised of 662 patients (Table 1 ), observed that the expression of serum vWF in liver cirrhosis with $\mathrm{PH}$ patients is significantly higher than that in liver cirrhosis without $\mathrm{PH}$ patients.

In addition, we used RevMan5.2 software for metaanalysis. In the case of no threshold effect, we combined SEN and SPE. The SEN of VWF diagnosis of PH was $82.3 \%$ and SPE was $78.2 \%$, indicating that the rate of missed diagnosis was $17.6 \%$ and the misdiagnosis rate was $21.8 \%$. +LR was $3.777(>1)$, indicating the possibility of PH when VWF was positive in the diagnosis of $\mathrm{PH}$; -LR was $0.22(<1)$, indicating that the possibility of $\mathrm{PH}$ cannot be excluded when the $v W F$ diagnosis of PH is negative. The forest map of DOR was drawn and heterogeneity analyzed. The values of $p=$ $0.1683(>0.1), I^{2}=40.6 \%(<50 \%)$ showed no heterogeneity. The odds ratio was 18.347 , suggesting that vWF has high accuracy in differentiating cirrhosis with or without $\mathrm{PH}$. Finally, the SROC curve was drawn. The AUC was 0.8896 and the $\mathrm{Q}$ index was 0.8203 , indicating that vWF has a high diagnostic efficiency for cirrhosis with $\mathrm{PH}$. Moreover, the results of this study showed that in the diagnosis of $\checkmark W F$, the variation range of SEN in the diagnosis of cirrhosis with $\mathrm{PH}$ was not significant $(0.788-0.855)$, suggesting that the stability of diagnosis was good.

In conclusion, this study suggests that serum vWF could be used as an effective and feasible method of noninvasive marker testing for diagnosis of liver cirrhosis with $\mathrm{PH}$. However, in this study, most of the research samples were too small; thus, serum vWF could not be found to evaluate the severity of liver cirrhosis with $\mathrm{PH}$. Further studies are still needed to evaluate the role of vWF levels in indicating the severity of liver cirrhosis with $\mathrm{PH}$.

\section{Acknowledgments}

This study was supported by the National Natural Science Foundation of China (No. 81760363), the National Key R\&D Program of China (No. 2016YFD0400605) and the Natural Science Foundation of Ningxia (NZ16156, 2018AAC02014).

\section{Conflict of interest}

The authors have no conflict of interests related to this publication. 


\section{Author contributions}

Contributed equally to this study (XCD, WLM, MKL), conceived the study, drafted the manuscript and critically revised it for important intellectual content (XCD, WLM, MKL, LNM), all authors read and approved the final manuscript.

\section{References}

[1] Gracia-Sancho J, Laviña B, Rodríguez-Vilarrupla A, García-Calderó H Fernández $\mathrm{M}$, Bosch $\mathrm{J}$, et al. Increased oxidative stress in cirrhotic rat livers: A potential mechanism contributing to reduced nitric oxide bioavailability. Hepatology 2008;47:1248-1256. doi: 10.1002/hep.22166.

[2] Groszmann RJ, Wongcharatrawee S. The hepatic venous pressure gradient: anything worth doing should be done right. Hepatology 2004;39:280-282. doi: $10.1002 /$ hep.20062.

[3] Reiberger T, Püspök A, Schoder $M$, Baumann-Durchschein $F$, Bucsics $T$, Datz $C$, et al. Austrian consensus guidelines on the management and treatment of portal hypertension (Billroth III). Wien Klin Wochenschr 2017;129: 135-158. doi: 10.1007/s00508-017-1262-3.

[4] Blann AD, Naqvi T, Waite M, McCollum CN. von Willebrand factor and endothelial damage in essential hypertension. J Hum Hypertens 1993;7:107-111.

[5] Ferro D, Quintarelli C, Lattuada A, Leo R, Alessandroni M, Mannucci PM, et al. High plasma levels of von Willebrand factor as a marker of endothelia perturbation in cirrhosis: relationship to endotoxemia. Hepatology 1996; 23:1377-1383. doi: 10.1002/hep.510230613.

[6] Hametner S, Ferlitsch A, Ferlitsch M, Etschmaier A, Schöfl R, Ziachehabi A, et al. The VITRO score (von Willebrand factor antigen/thrombocyte ratio) as a new marker for clinically significant portal hypertension in comparison to other non-invasive parameters of fibrosis including ELF test. PLoS One 2016; 11:e0149230. doi: 10.1371/journal.pone.0149230.
[7] La Mura V, Reverter JC, Flores-Arroyo A, Raffa S, Reverter E, Seijo S, et al. Von Willebrand factor levels predict clinical outcome in patients with cirrhosis and portal hypertension. Gut 2011;60:1133-1138. doi: 10.1136/gut.2010. 235689.

[8] Nomura S, Hisashige A, Yoneoka D, Kanda M, Miyamoto K, Segawa M, et al. An assessment of the characteristics and quality of diagnostic accuracy studies for positron emission tomography conducted in Japan: a systematic review. EJNMMI Res 2015;5:6. doi: 10.1186/s13550-0150084-4.

[9] Wu H, Yan S, Wang G, Cui S, Zhang C, Zhu Q. von Willebrand factor as a novel noninvasive predictor of portal hypertension and esophageal varices in hepatitis B patients with cirrhosis. Scand J Gastroenterol 2015;50: 1160-1169. doi: 10.3109/00365521.2015.1037346.

[10] Ferlitsch M, Reiberger T, Hoke M, Salzl P, Schwengerer B, Ulbrich G, et al. von Willebrand factor as new noninvasive predictor of portal hypertension, decompensation and mortality in patients with liver cirrhosis. Hepatology 2012;56:1439-1447. doi: 10.1002/hep.25806.

[11] Homoncik M, Ferlitsch A, Reiberger T, Ulbrich G, Gangl A, Peck-Radosavljevic $M$. Von Willebrand factor predicts clinically significant portal hypertension in patients with liver cirrhosis. J Hepatol 2008;48:S114-S115. doi: 10. 1016/s0168-8278(08)60288-3.

[12] Zamora J, Abraira V, Muriel A, Khan K, Coomarasamy A. Meta-DiSc: a software for meta-analysis of test accuracy data. BMC Med Res Methodol 2006 6:31. doi: 10.1186/1471-2288-6-31.

[13] Gouya H, Grabar S, Vignaux O, Saade A, Pol S, Legmann P, et al. Portal hypertension in patients with cirrhosis: indirect assessment of hepatic venous pressure gradient by measuring azygos flow with 2D-cine phasecontrast magnetic resonance imaging. Eur Radiol 2016;26:1981-1990. doi: 10.1007/s00330-015-3991-x.

[14] Abdelmoneim SS, Talwalkar J, Sethi S, Kamath P, Fathalla MM, Kipp BR, et al. A prospective pilot study of circulating endothelial cells as a potential new biomarker in portal hypertension. Liver Int 2010;30:191-197. doi: $10.1111 / \mathrm{j} .1478-3231.2009 .02132 . x$. 